\title{
The Role of F18-FLT PET / CT in Assessing Early Response to TARE and TACE in Patients with Primary and Metastatic Liver Tumors
}

DEMET NAK ( $\sim$ demetnak@yahoo.com )

Rize Recep Tayyip Erdogan Training and Research Hospital https://orcid.org/0000-0002-9756-7788 Nuriye Ozlem Kucuk

Ankara University Faculty of Medicine

Emre Can Celebioglu

Ankara University Faculty of Medicine

Mehmet Sadik BILGIC

Ankara University Faculty of Medicine

Serhat HAYME

Ankara University Faculty of Medicine

Metin Kemal KIR

Ankara University Faculty of Medicine

\section{Research Article}

Keywords: liver tumors, chemoembolization, radioembolization, early response, F18-FLT PET/CT

Posted Date: February 25th, 2021

DOI: https://doi.org/10.21203/rs.3.rs-238191/v1

License: (1) This work is licensed under a Creative Commons Attribution 4.0 International License.

Read Full License 


\section{Abstract}

Objective

This study aims to identify F18-FLT PET / CT's role in the assessment of early response to intraarterial chemoembolization and radioembolization treatments in patients with unresectable primary and metastatic liver tumors.

Methods

This single-center study included 63 patients who underwent F18-FLT PET / CT for response evaluation after TACE and TARE treatments. After excluding 20 patients due to various reasons, 43 patients were analyzed. The compatibility of change in semi-quantitative values obtained from the F18-FLT PET / CT images with the treatment responses detected in F18-FDG PET / CT, CT, and MR images was evaluated.

Results

There was no correlation between early metabolic, morphological response, F18-FLT visual change, $\triangle S U V m a x, \Delta S U V m e a n$, and $\Delta S U V p e a k$ values. There was no significant correlation between F18-FLT visual change, $\Delta S U V \max , \Delta S U V$ mean, $\Delta S U V$ peak, and OS, PFS for the target lobe PFS for the whole-body. The survival distributions for the patients with and without $>30 \%$ change in $\Delta$ SUVmax and $\Delta$ SUVpeak values were statistically significantly different $(p<.009$ and $p<.024$, respectively).

Conclusion

It has not been demonstrated that F18-FLT PET / CT imaging is one of the methodologies that should be applied primarily in monitoring early response to embolization treatment in patients with unresectable primary and metastatic liver tumors.

\section{Introduction}

Both metastases and primary malignancies, such as hepatocellular carcinoma (HCC) and cholangiocellular cancer, are common in the liver. Metastases are the most common liver malignancy, and leading tumors that metastasize to the liver are colorectal cancer, neuroendocrine tumors, other gastrointestinal cancers, and breast cancer. HCC is the sixth common cause of cancer and the third common cause of cancer-related deaths worldwide [1,2]. Since liver involvement is effective on survival, curative surgical applications are the first-line therapy, either together with adjuvant chemotherapy or alone, providing the most significant survival advantage. However, surgery cannot be applied to most patients at diagnosis or tumor recurrence due to advanced-stage disease or inappropriate clinical status $[2,3]$. Local ablative applications such as radiofrequency (RFA), microwave (MWA) and cryo-ablation, irreversible electroporation (IRE), endovascular transarterial chemoembolization (TACE), and transarterial radioembolization (TARE) provide minimally invasive and safe treatment [4-11]. It has been reported that 
TARE and TACE provide a long-term survival advantage and low toxicity, especially in patients with good performance and low tumor burden [7-11].

The prediction or early detection of response to therapy prevents unnecessary toxicity and cost that may be life-threatening in non-responder patients and provides earlier use of other treatment options that may be effective. Morphological response evaluation with CT and MR requires a relatively long period and tumor shrinkage. PET/CT or PET/MR hybrid imaging, based on metabolic processes, provides earlier response assessment and concurrent anatomical information. F18-FDG is the most commonly used agent in PET imaging [12-16]. However, since tumors with low-glucose metabolism and low cellularity, small-sized and well-differentiated tumors show low FDG uptake, alternative agents such as thymidine analog 3 '-deoksy-[18F]-3'-fluorotimidin ( ${ }^{18} \mathrm{~F}$-FLT], which reflects the proliferation of cells, ${ }^{11} \mathrm{C}$-acetate, which reflects hypoxia, ${ }^{11} \mathrm{C}$-choline or ${ }^{18} \mathrm{~F}$-choline, which reflects aerobic metabolism (fatty acid synthesis) are being investigated [7-22,25-27].

F18-FLT, an analog of thymidine, is phosphorylated with thymidine kinase-1 (TK1) and is converted to F18-FLT-monophosphate, which can not penetrate DNA and is trapped in the cytosol. F18-FLT is a TK1specific substrate that increases in proliferating cells while not found in silent cells and correlates with a proliferation marker Ki-67 index [22,23]. Imaging with F18-FLT has advantages such as non-invasive quantitation of cell proliferation, three-dimensional tumor imaging, and evaluating the whole tumor proliferation heterogeneity in multiple tumor areas simultaneously. Studies show that tumor proliferation changes can be detected early with F18-FLT PET / CT after radiotherapy [22-27]. Knowing that TARE is an internal radiotherapy method, this study aims to describe F18-FLT PET / CT's role in assessing early response to TARE in patients with primary and metastatic liver tumors.

\section{Materials And Methods}

Ethics committee approval (13-117-19) was obtained for this single-center study with prospective and retrospective components. The study was performed under the Helsinki Directive and Good Clinical Practices Guidelines. Informed consent was obtained from all volunteers included in the study.

\section{Patients:}

This study included the patients who underwent TACE and TARE treatment for histologically / cytologically or radiologically diagnosed primary (HCC, cholangiocellular carcinoma, etc.) or metastatic liver tumor; staged with CT, MR, and F18-FDG PET / CT or PET / MR imaging; has Eastern Cooperative Oncology Group (ECOG) performance score $\leq 2$; was over 18 years old; were found suitable for TARE and TACE treatments; were followed for more than three months; had available data; gave informed consent to participate.

Patients with severe pain and claustrophobia, etc., preventing imaging; ECOG performance score> 2; patients who left the follow-up before the 3rd month; whose data are not available; who did not want to participate in the study were excluded. 
There was no intervention in the treatment selection or management of the patients. The relevant specialist chose the treatment according to the standard evaluations.

\section{F18-FLT PET / CT imaging protocols and evaluation:}

The presence or history of systemic or local ablative therapy, chronic disease, etc. that can affect the evaluation was questioned and noted. Oral hydration and bladder emptying before imaging was provided to reduce the total body radiation dose and increase the image quality. Approximately 60 minutes after the F18-FLT were given intravenously, the whole-body PET / CT images were taken. Following CT for attenuation correction, and anatomical correlation, whole-body PET images were obtained, in the supine position, from the vertex to the middle of the thigh, and 3 min per bed. PET /CT Discovery ST (GE Healthcare Waukesha, Wisconsin, USA) was used for PET hybrid imaging. After assessing maximum intensity projection (MIP), cross-sectional, and fusion images, areas with high (hyper-metabolic), heterogeneous (mixed), similar (iso-metabolic), and low (hypo-metabolic) uptake from adjacent liver parenchyma were noted.

F18-FLT PET / CT was performed twice: as a baseline before treatment and for response evaluation after treatment. Appearance and SUVmax, SUVmean, SUVpeak values of the target lesion-defined as sole or the most extensive lesion in the target lobe were noted. Since the reference parenchymal SUV values of the patients showed a significant difference both between the patients and the pre-and post-treatment images of the same patient, the target background ratio (TBR) of the target lesions were calculated by proportioning the SUV values of the target lesion to reference values and were evaluated separately. Patients were divided into groups with and without the change of SUV values calculated by the difference between the target lesion's post-treatment and pre-treatment SUV values, which were calculated and referred to as delta $(\Delta)$ SUV values.

\section{Statistical analysis:}

The changes between baseline and post-treatment F18-FLT PET / CT images were compared to the responses detected with F18-FDG PET / CT and CT/MRI, evaluated according to the PERCIST and RECIST 1.1 criteria, respectively, and progression-free survival and overall survival. All statistical analyses were performed using IBM SPSS for Windows version 15.0 (SPSS, Chicago, IL, USA). Shapiro-Wilks and Kolmogorov-Smirnov test was used to assess the assumption of normality. The continuous variables that do not have normal distribution were expressed as median (minimum-maximum). For non-normallydistributed continuous variables, differences between groups were tested using Mann Whitney U-test and Kruskal Wallis test. Lastly, Pearson chi-square analysis and Fisher's exact test determined associations between categorical variables, while Pearson and Spearman correlation analysis specified associations between continuous variables. The survival times of groups were obtained with Kaplan Meier analysis, and the difference between groups in survival times compared with the Log Rank test. A two-sided pvalue $<0.05$ was considered statistically significant. 


\section{Findings}

\section{Patients}

Sixty-three consecutive patients were included in the study between December 2018 and January 2020, who underwent pre-and post-treatment F18-FLT PET/CT to evaluate response to TARE and TACE treatments. Although all patients underwent baseline imaging, three of the TACE-receiving patients and sixteen of the TARE-receiving patients could not undergo F18-FLT PET/CT or other imaging for response evaluation either due to decreased performance status that hindered further procedure or death.

Three patients who received TACE did not undergo PET/CT or MR/CT imaging to evaluate response to treatment, and most of their data were missing. Only one TACE-receiving patient who underwent all imagings and survived more than six months had available data. This one TACE-receiving patient excluded from analysis, and 43 TARE-receiving patients were analyzed to have a homogenous population and statistical analysis.

30/43 patients were male, and the median age was 63 ( $\min 38$ - $\max 79)$ years. Twenty-four patients had a primary liver tumor, and 14, 2, 2, 1 patient had liver metastasis from the colon, gastric, breast, and pancreas cancer, respectively. Detailed patient characteristics are listed in Table 1. One patient did not undergo F18-FDG PET/CT scanning for response evaluation because of the tumor's non-FDG avidity at the baseline. The remaining patients completed both F18-FLT and F18-FDG PET/CT scannings. Morphological response evaluation was done with contrast-enhanced CT for two patients, and with contrast-enhanced MRI for 41 patients.

\section{Metabolic and morphological assessment}

43 patients underwent F18-FDG PET/CT median 15 ( $\min 1$ - $\max 64)$ days before TARE and 42 patients underwent F18-FDG PET/CT median 47 ( $\min 34-\max 72$ ) days after treatment. Five patients had complete, 13 patients had a partial response in early metabolic response, while 14 had stable, and 10 had progressive metabolic disease on target lobe/tumor with F18-FDG PET/CT.

Forty-three patients underwent CT/MRI median 13 (min 1- max 79) days before and median 97 (min 46max 171) days after TARE. 2 patients had complete, five patients had a partial response, while 17 had stable, and 17 had progressive disease on contrast-enhanced MR, while both two patients, who underwent contrast-enhanced CT, had a partial response.

\section{F18-FLT PET/CT}

Administered activities were median 238 ( $\min 170.6-\max 384) \mathrm{MBq}$ for pre-treatment and median 238 (min 126- $m a x 345$ ) MBq for post-treatment F18-FLT PET/CT imaging. At baseline F18-FLT PET/CT, there were 6 hyper-metabolic, 5 mixed, 13 iso- and 20 hypo-metabolic target lesions. There was 1 hypermetabolic, 2 mixed, 8 iso- and 22 hypo-metabolic target lesions on post-treatment F18-FLT PET/CT. 2/6 of the hypermetabolic lesions remained, while 1/6 became mixed, 1/6 became iso- and 2/6 became hypo- 
metabolic. $1 / 5$ of the target lesions with mixed appearance persisted; the rest majority became hypometabolic. $1 / 9$ iso-metabolic lesion became hypo-metabolic while the remaining $8 / 9$ lesions remained. All hypo-metabolic lesions persisted. PET/CT and contrast-enhanced liver MR images of the patient with persistent hypermetabolic lesions and progressive disease are presented in Figure 1.

There was no correlation between the diagnosis, longest diameter of the target lesion, volume and percent of tumor in the target lobe, age, the number of lesions in the target lobe, early metabolic, morphological response and F18-FLT visual change, $\triangle$ SUVmax, $\triangle S U V$ mean, $\Delta S U V$ peak, $\Delta S U V$ maxTBR, $\triangle S U V m e a n T B R$, and $\triangle S U V$ peakTBR values. Calculated $p$ values from statistical analyses are given in Table 3.

\section{Survival}

During 18.4 months follow-up, 22 patients died, 27/43 (63\%) patients had progressive disease on the target lobe, 32 had disease progression, and 11 were on follow-up without recurrence. Overall survival (OS) was median 7.0 ( $\min 3.3-$ max 17.4) months, progression-free survival (PFS) was median 3.4 (min 1.3-max 17.4) months for target lobe; and median 3.2 ( $\min 1.3$-max 17.4) months for whole-body.

There was no significant correlation between F18-FLT visual change, $\Delta S U V m a x, \Delta S U V$ mean, $\Delta S U V$ peak, $\triangle$ SUVmaxTBR, $\triangle$ SUVmeanTBR, and $\triangle$ SUVpeakTBR and OS, PFS for target lobe and PFS for whole-body (Table 3). A log-rank test was run to determine if there were differences in the target lobe's progressionfree survival distribution for the $\Delta S U V \max$ and $\Delta$ SUVpeak groups when cut-off $>30 \%$ change was applied. The survival distributions for the patients with and without a change in $\Delta S U V$ max were statistically significantly different, $\chi 2(2)=6,774, p<.009$. The survival distributions for the patients with and without $>30 \%$ change in $\Delta$ SUVpeak were also statistically significantly different, $\chi 2(2)=5,095, p<$ .024. Statistical analysis could not provide a median value and estimated survival chance at 209. day was 0,549 $\pm 0,129$ STD for 17 patients with no change in SUVmax, while the estimated survival chance at 92. day was $0,500 \pm 0,098$ STD in patients with $>30 \%$ change in SUVmax. Estimated survival proportion at 209. day was $0,514 \pm 0,134$ in 16 patients without change in SUVpeak value; while this proportion was $0,519 \pm 0,096$ at 90 .day (Figure 2, Table 3).

\section{Discussion}

Both metastases and primary malignancies are commonly seen in the liver. As liver involvement is effective on survival, early detection of response has a crucial role in patient management (2-5). After LRTs such as TARE and TACE, there is no standard response evaluation and follow-up protocol; valuation is performed at different times with PET / CT, CT, or MR, depending on the center's practice and patient management. Since response assessment with CT and MRI takes a longer time and have their limitations, PET, a functional and molecular imaging technique, is used for early response evaluation with agents that reflect tumor-specific metabolism. Providing metabolic and anatomical information, PET / CT, and PET / MR have become the leading imaging techniques in cancer patient management [12-22]. 
F18-FDG PET / CT is the most common metabolic imaging method in diagnosing, staging, restaging and evaluating the treatment response due to increased glucose metabolism in many types of cancer. F18FDG PET-CT can be used to assess treatment response in poorly differentiated and high-grade tumors. However, since small and well-differentiated tumors (such as HCC, NET) shows low or no FDG uptake due to low glucose metabolism and cellularity, imaging with new tumor-specific agents is needed [17-22,2527]. PET / CT imaging with F18-FLT reflects cells' proliferation, is a non-invasive method in a personalized treatment approach [25-27].

In addition to complex and competing factors in the FLT uptake mechanism, there are notable differences between patient preparation, imaging time after injection, protocol, amount of injected activity, reconstruction method, timing before and after treatment, and patient numbers in studies withF18-FLT PET / CT. The power of studies is weak, as the number of patients achieving all the treatments and an ideal comparison in different disease groups is limited. It is noteworthy that data and various analysis techniques, such as semi-quantitative or visual evaluation are used [22-30]. Considering all the factors mentioned above are influential in the response evaluation, and there is no standard protocol, it is difficult to compare the studies.

As far as it is known, this is the first study to investigate the place of F18-FLT PET / CT in the early evaluation after TARE. Mogensen et al. investigated F18-FLT PET/CT's role in patients with at least one measurable $(>1 \mathrm{~cm}$ ) colorectal cancer liver metastasis and received first-line chemotherapy. They reported a reduction in F18-FLT uptake in 23 patients (85\%), whereas there was no relationship between the early change in measured F18-FLT SUVmax, and RECIST 1.1 based response $(p=0.24)$ [31]. There was no relationship between $\triangle S U V m a x, \triangle S U V m e a n$, and $\triangle S U V$ peak values and RECIST 1.1 and PERCIST based responses in this current study. However, there was a significant relationship between PFS for target lobe and change in SUVmax and SUVpeak values.

Contractor K. et al. investigated F18-FLT PET/CT's role in evaluating the treatment response of breast and colorectal cancer liver metastases. They reported that 26/33 metastases were visible after kinetic spatial filtering (FLT-PETKSF). FLT-PET SUVave or SUVmax and FLT-PETKSF showed a significant decrease in responders two weeks after the first-line chemotherapy. It has been reported that the change in FLT can distinguish those who responded to the treatment with $83 \%$ sensitivity and $78 \%$ specificity from nonresponders [30]. In this current study, F18-FDG and F18-FLT PET / CT were evaluated for the treatment response after TARE, an LRT, not a systemic treatment. There was no significant correlation between $\triangle S U V m a x, \triangle S U V m e a n$, and $\triangle S U V p e a k$ values among the patients with and without response; therefore, sensitivity and specificity could not be calculated.

The critical point in the early evaluation of the treatment response is to distinguish non-responder to discontinue unnecessary treatment, thus avoid toxicity and cost. It is essential to determine the resectable disease from those that require more aggressive treatment. Patients with shrinkage of tumors up to $30 \%$ are considered to have stable disease, according to RECIST 1.1, and unresponsive to treatment [12-17]. In this study, tumor sizes of patients with stable disease decreased, reflecting the beneficial effect of 
treatment. However, since this decrease in size remained below the RECIST 1.1 criteria, it was accepted as a stable disease and unresponsive to treatment. It should be recognized that patients with stable disease, especially with colon cancer, are admitted as responders and continue to receive systemic therapy in clinical practice [32]. Generally, chemotherapy-refractory liver metastases are referred for LRTs such as TARE. Thus, even defined stable disease can provide more prolonged survival and can be accepted as responsive. If patients with stable disease are admitted as responders to therapy, statistical analysis can be found significant in long-term follow-up results. Since there was no increase in the F18-FLT uptake after treatment in any of the patients, it could not be evaluated whether this was related to progressive disease. Since the tumors were hypometabolic in most patients, the changes in the F18-FLT SUVmax, SUVmean, and SUVpeak values were not significantly different in responder and non-responder patients. Since liver resection was not performed on any of the patients after embolization, except for the transplantation patient, post-treatment histopathological tumor changes and correlation with F18-FLT values could not be evaluated.

The most significant limitation of this study is the small sample size, consequent heterogeneous patient population, and the small number of patients who responded to the therapy. Therefore, in statistical analysis, results reaching a significant degree could not be obtained in SUV parameters. TARE candidate patients have many different clinical scenarios such as highly variable liver lesion number and size, disease stage, history of single or multi-step systemic treatment, liver resection, transplant, and LRT's. Also, since there is a clear difference in disease etiologies, current clinical and radiological status, it was impossible to standardize the patient group. Further studies with larger and standardized patient populations are needed.

It can be argued that the timing of the F18-FLT was not right. TARE is an internal radiotherapy procedure, and response to radiotherapy is generally evaluated later than chemotherapy/ selective systemic therapies [25,26]. F18-FDG PET/CT and F18-FLT PET/CT imaging were done $\geq 4$ weeks after the procedure. Studies evaluating radiotherapy response revealed a significant relationship between F18-FLT PET/CT and response or survival in patients with head-neck, esophageal, breast, lung, rectal, etc. cancer [26]. This study aimed to distinguish real responders from non-responders who were grouped based on post-radiotherapy response assessment techniques F18-FDG PET/CT and CT or MR. No correlation was found between the semi-quantitative values such as $\triangle S U V \max , \triangle S U V$ mean, $\triangle$ SUVpeak, SUVmaxTBR, SUVmeanTBR, and SUVpeakTBR values calculated from F18-FLT PET / CT images. There was a significant relationship between OS and $\Delta S U V \max$, and $\triangle S U V$ peak values only when $>30 \%$ change accepted as significant.

\section{Conclusion}

It has not been shown a significant relationship between the changes in F18-FLT PET/CT SUVmax, SUVmean, SUVpeak, SUVmaxTBR, SUVmeanTBR, and SUVpeak values with neither with the early metabolic response in F18-FDG PET/CT nor with the morphological response in contrast-enhanced CT or MR in patients with primary and metastatic unresectable liver tumor undergoing embolization therapy. 
There was a significant relationship between OS and $\Delta S U V \max$, and $\Delta S U V$ peak values when $>30 \%$ change accepted as significant. Reproducible and re-applicable clinical data from a larger and standardized patient population is required to assess the role of F18-FLT PET-CT in the evaluation of response to TARE treatment. Although the clinical use of F18-FLT PET / CT is limited, it can be used as an alternative/complementary to F18-FDG PET / CT in the early evaluation of the treatment response in patients undergoing TARE for primary or secondary liver tumor.

\section{Declarations}

Funding: Author's own work.

Conflicts of interest: All authors declare no conflict of interest.

\section{References}

1. Parkin DM, Bray F, Ferlay J, Pisani P. Global cancer statistics, 2002. CA Cancer J Clin. 2005;55(2):74108. doi: 10.3322/canjclin.55.2.74. PMID: 15761078.

2. Bosch FX, Ribes J, Diaz M, Cleries R. Primary Liver cancer: worldwide incidence and trends. Gastroenterology. 2004;127:5-16. doi: 1053/j.gastro.2004.09.011

3. Yao FY, Bass NM, Nikolai B, et al. A follow-up analysis of the pattern and predictors of dropout from the waiting list for liver transplantation in patients with hepatocellular carcinoma: implications for the current organ allocation policy. Liver Transpl, 2003;9: 684-692. doi:1053/jlts.2003.50147

4. Ryan MJ, Willatt J, Majdalany BS, et al. Ablation techniques for primary and metastatic liver tumors. World J Hepatol. 2016;8:191-199. doi:10.4254/wjh.v8.i3.191

5. Memon K, Lewandowski RJ, Kulik L, Riaz A, Mulcahy MF, Salem R. Radioembolization for primary and metastatic liver cancer. Seminars in Radiation Oncology. 2011;21:294-302. DOI: 10.1016/j.semradonc.2011.05.004.

6. Harding JJ, Abou-Alfa GK. Treating advanced hepatocellular carcinoma: how to get out of first gear. Cancer, 2014;120:3122-3130. doi:1002/cncr.28850

7. Salem R, Lewandowski RJ, Kulik L, et al. Radioembolization results in longer time-to-progression and reduced toxicity compared with chemoembolization in patients with hepatocellular carcinoma. Gastroenterology. 2011;140:497-507. doi:10.1053/j.gastro.2010.10.049

8. Lance C, McLennan G, Obuchowski N, et al. Comparative analysis of the safety and efficacy of transcatheter arterial chemoembolization and yttrium-90 radioembolization in patients with unresectable hepatocellular carcinoma. J Vasc Interv Radiol 2011; 22:1697-1705. doi: 10.1016/j.jvir.2011.08.013.

9. Kooby DA, Egnatashvili V, Srinivasan S, et al. Comparison of yttrium-90 radioembolization and transcatheter arterial chemoembolization for the treatment of unresectable hepatocellular carcinoma. J Vasc Interv Radiol. 2010; 21:224-230. doi:10.1016/j.jvir.2009.10.013. 
10. Carr BI, Kondragunta V, Buch SC, Branch RA. Therapeutic equivalence in survival for hepatic arterial chemoembolization and yttrium 90 microsphere treatments in unresectable hepatocellular carcinoma: a Two-Cohort Study. Cancer. 2011;116:1305-1314.

11. Lewandowski RJ, Kulik LM, Riaz A, et al. A comparative analysis of transarterial downstaging for hepatocellular carcinoma: chemoembolization versus radioembolization. Am J Transplant. 2009; 9:1920-1928

12. Therasse $P$, Arbuck SG, Eisenhauer EA, et al. New guidelines to evaluate the response to treatment in solid tumors. Journal of the National Cancer Institute. 2000;92:205-216. doi:10.1093/jnci/92.3.205

13. Forner $A$, Ayuso $C$, Varela $M$, et al. Evaluation of tumor response after locoregional therapies in hepatocellular carcinoma: are response evaluation criteria in solid tumors reliable. Cancer. 2009; 115:616-623.

14. Miller FH, Keppke AL, Reddy $D$, et al. response of liver metastasis after treatment with yttrium-90 microspheres: role of size, necrosis and PET. AJR Am J Roentgenol 2007; 188: 776-783.

15. Wong CY, Salem R, Raman S, Gates VL, Dworkin HJ. Evaluating 90Y-glass microsphere treatment response on unresectable colorectal liver metastases by (F18) FDG PET: a comparison with CT or MRI. Eur J Nucl Med Mol Imaging 2002; 29:815-820. doi: 10.1007/s00259-002-0787-4.

16. Singh P, Anil G: Yttrium-90 radioembolization of liver tumors: what do the images tell us? Cancer Imaging. 2014, 13:645-657. doi:10.1102/1470-7330.2013.0057

17. Park JW, Kim JH, Kim SK et al. A prospective evaluation of F18-FDG and 11C-acetate PET/CT for detection of primary and metastatic hepatocellular carcinoma. Nucl. Med. 2008; 49, 1912-1921.doi: 10.2967/jnumed.108.055087.

18. Ho CL, Chen S, Yeung DW, Cheng TK. Dual-tracer PET/CT imaging in evaluation of metastatic hepatocellular carcinoma. Nucl.Med. 2007;48, 902-909.

19. Dubash SR, Idowu $O A$, Sharma R. The emerging role of positron emission tomography in hepatocellular carcinoma. Hepatic Oncology. 2015;2:1-10. doi:10.2217/HEP15.6

20. Sacks A, Peller PJ, Surasi DS, Chatburn L, Mercier G, Subramaniam RM. Value of PET/CT in the management of liver metastases, Part 1. AJR Am J Roentgenol 2011; 197: 256-259.

21. Johnbeck CB, Knigge U, Kjær A. PET tracers for somatostatin receptor imaging of neuroendocrine tumors: current status and review of the literature. Future Oncol. 2014;10:2259-2277. doi: 2217/FON.14.139

22. Shields AF, Grierson JR, Dohmen BM et al. Imaging proliferation in vivo with (F-18)FLT and positron emission tomography. Med. 1998;4, 1334-1336. doi: 10.1038/3337.

23. Chalkidou A, Landau DB, Odell EW, Cornelius VR, O'Doherty MJ, Marsden PK. Correlation between Ki67 immunohistochemistry and F18-Fluorothymidine uptake in patients with cancer: A systematic review and meta- Eur J Cancer 2012;48:3499-513. doi: 10.1016/j.ejca.2012.05.001

24. Krohn KA, Mankoff DA, Eary JF. Imaging cellular proliferation as a measure of response to therapy. J Clin Pharmacol 2001;41:96- 
25. Peck M, Pollack HA, Friesen A, et al.Applications of PET imaging with the proliferation marker (F18)FLT. Q J Nucl Med Mol Imaging. 2015;59:95-104.

26. Sanghera $B$, Wong WL, Sonoda $\mathrm{LI}$, et al. FLT PET-CT in evaluation of treatment response. Indian $\mathrm{J}$ Nucl Med. 2014;29:65-73. doi:10.4103/0972-3919.130274

27. Eckel F., Herrmann K., Schmidt S. et al. Imaging of Proliferation in Hepatocellular Carcinoma with the In Vivo Marker F18-Fluorothymidine. J Nucl Med 2009; 50:1441-1447 doi:

10.2967/jnumed.109.065896

28. Flexner, C., van der Horst, C., Jacobson, M.A., et al., Relationship between plasma concentrations of $3^{\prime}$ - fluoro-3' -deoxythymidine (Alovudine) and antiretroviral activity in two concentration-controlled trials. J. Infect. Dis. 1994;170: 1394-1403.

29. Muzi, M., Vesselle, H., Grierson, J.R., et al. Kinetic analysis of $3^{\prime}$ - fluoro-3' -deoxythymidine PET studies: validation studies in patients with lung cancer. J. Nucl. Med. 2005; 46: 274-282.

30. Contractor K., Challapalli A., Tomasi G. et al. Imaging of cellular proliferation in liver metastasis by (F18)fluorothymidine positron emission tomography: Effect of therapy. Physics in Medicine\&Biology.2012;57:3419-33.doi:10.1088/0031-9155/57/11/3419.

31. Mogensen MB, Loft A, Aznar M, et al. FLT-PET for early response evaluation of colorectal cancer patients with liver metastases: a prospective study. EJNMMI Research. 2017;7:56. doi:

10.1186/s13550-017-0302-3

32. Chibaudel B, Maindrault-Goebel F, Lledo G, et al. Can chemotherapy be discontinued in unresectable metastatic colorectal cancer? The GERCOR OPTIMOX2 Study. J Clin Oncol. 2009;27:5727-33. doi: 10.1200/JCO.2009.23.4344.

\section{Tables}

Table 1. Patient characteristics 


\section{Characteristics}

Median (Min.-Max.)

$\mathrm{N}=43$

$\%$

Gender

Male

30

70

Female

13

30

Age

63 (38- 79) years

\section{Underlying liver disease}

Yes

15

No

28

65

\section{Previous treatments}

Surgery

RFA

TACE

TARE

Chemotherapy alone

Chemotherapy+LRT

Microsphere

Resine

$0,65(0,6-1,3) \mathrm{GBq}$

6

14

Glass

6,5 (3- 18) GBq

37

Target lobe

Right

34

79

Left

8

Transplanted liver

1

2

\section{Primary tumor}

HCC

Klatskin

7

Colon

14

33

Gastric

2

4,6

Breast

2

4,6




\begin{tabular}{|c|c|c|c|}
\hline Pancreas & & 1 & 2 \\
\hline \multicolumn{4}{|l|}{ Presence of primary tumor } \\
\hline Yes & & 27 & 63 \\
\hline $\mathrm{HCC}$ & & 17 & 40 \\
\hline Klatskin & & 7 & 16 \\
\hline Colon & & 3 & 7 \\
\hline No & & 16 & 37 \\
\hline \multicolumn{4}{|l|}{ Extrahepatic metastases } \\
\hline Yes & & 20 & 46,5 \\
\hline No & & 23 & 53,5 \\
\hline \multicolumn{4}{|l|}{ The largest diameter of target lesion } \\
\hline pretreatment & $49,7(8-190) \mathrm{mm}$ & & \\
\hline posttreatment & $60,3(9-190) \mathrm{mm}$ & & \\
\hline \multicolumn{4}{|l|}{ Number of lesions on target lobe } \\
\hline 1 & & 11 & 26 \\
\hline $1-5$ & & 11 & 26 \\
\hline $5-10$ & & 7 & 16 \\
\hline$>10$ & & 14 & 32 \\
\hline Tumor volume percent on target lobe & $14(1-100) \%$ & & \\
\hline \multicolumn{4}{|l|}{ Event after TARE } \\
\hline Alive- disease progression & & 13 & 30 \\
\hline Alive-partial response or stable disease & & 8 & 19 \\
\hline Died due to disease progression/other causes & & 14 & 33 \\
\hline Died due to liver failure & & 8 & 19 \\
\hline \multicolumn{4}{|l|}{ Visual change on 18F-FLT PET/CT after TARE } \\
\hline Yes & 24 & 55,8 & \\
\hline No & 19 & 44,2 & \\
\hline $\begin{array}{l}\text { HCC: hepatocellular carcinoma; LRT: locoregio } \\
\text { transarterial chemoembolization; TARE: transa }\end{array}$ & $\begin{array}{l}\text {; RFA; radiofrequen } \\
\text { jembolization. }\end{array}$ & ion; TAC & \\
\hline
\end{tabular}


SUV value

\section{Pre-treatment 18F-FLT PET/CT}

$\mathrm{SUV}_{\max }$

SUV $_{\text {mean }}$

SUV $_{\text {peak }}$

SUV $_{\text {max }}$ TBR

$\mathrm{SUV}_{\text {mean }} \mathrm{TBR}$

SUV $_{\text {peak }}$ TBR

\section{Post-treatment 18F-FLT PET/CT}

SUV $_{\text {max }}$

$\mathrm{SUV}_{\text {mean }}$

SUV $_{\text {peak }}$

SUV $_{\text {max }}$ TBR

SUV $_{\text {mean }}$ TBR

SUV $_{\text {peak }}$ TBR
Median (Min.-Max.)

$6,7(2,7-22) \mathrm{g} / \mathrm{mL}$

$4,4(1,1-12,4) \mathrm{g} / \mathrm{mL}$

$4,9(1-18,2) \mathrm{g} / \mathrm{mL}$

$0,9(0,3-3,0)$

$0,8(0,2-2,4)$

$0,9(0,1-3,2)$

$3,6(0,9-14,9) \mathrm{g} / \mathrm{mL}$

$4,9(1-26,5) \mathrm{g} / \mathrm{mL}$

$0,7(0,3-3,8)$

$0,6(0,1-3,3)$

$0,7(0,1-4,1)$

Difference between pre- and post-treatment 18F-FLT values

$\triangle \mathrm{SUV}_{\max }$

$\triangle \mathrm{SUV}_{\text {mean }}$

$\triangle \mathrm{SUV}_{\text {peak }}$

$\triangle \mathrm{SUV}_{\max } \mathrm{TBR}^{\mathrm{T}}$

$\triangle \mathrm{SUV}_{\text {mean }} \mathrm{TBR}$

$\triangle$ SUV $_{\text {peak }} T B R$
$-2,0(-9,3-25,2)$

$0,9(-8,1-17,1)$

$-2,0(-8,2-21,2)$

$-1,0(-1,3-0,8)$

$0(-1,5-0,9)$

$0(-1,4-1,0)$

18F-FLT: Fluorothymidine; SUV $_{\text {max }}$ : maximum standard uptake value; SUV $_{\text {mean }}$ : mean standard

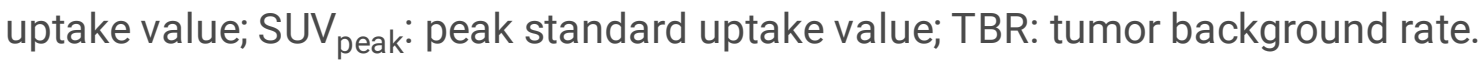




\section{p-value}

Visual change-early response

$0,93^{\mathrm{a}}$

Visual change-anatomical response

$0,71^{\mathrm{a}}$

\section{F-FLT SUV values-early response}

\begin{tabular}{|c|c|}
\hline$\triangle \mathrm{SUV}_{\max }$ & $0,29^{a}$ \\
\hline$\Delta S U V_{\text {mean }}$ & $0,1^{\mathrm{b}}$ \\
\hline$\Delta S U V_{\text {peak }}$ & $0,43^{a}$ \\
\hline$\triangle \mathrm{SUV}_{\max } \mathrm{TBR}^{\mathrm{T}}$ & $0,6^{\mathrm{a}}$ \\
\hline$\Delta S U V_{\text {mean }} T B R$ & $0,27^{a}$ \\
\hline$\Delta \mathrm{SUV}_{\text {peak }} \mathrm{TBR}$ & $0,28^{a}$ \\
\hline
\end{tabular}

\section{F-FLT SUV values-anatomical response}

$\begin{array}{lc}\triangle \mathrm{SUV}_{\text {max }} & 0,45^{\mathrm{b}} \\ \Delta \mathrm{SUV}_{\text {mean }} & 0,66^{\mathrm{c}} \\ \Delta \mathrm{SUV}_{\text {peak }} & 0,45^{\mathrm{b}} \\ \Delta S U V_{\text {max }} T B R & 0,4^{\mathrm{b}} \\ \triangle \mathrm{SUV}_{\text {mean }} T B R & 0,4^{\mathrm{b}} \\ \triangle \mathrm{SUV}_{\text {peak }} \text { TBR } & 0,4^{\mathrm{a}}\end{array}$

Overall Survival-SUV values, visual change

$\Delta \mathrm{SUV}_{\max }$

$0,63^{\mathrm{C}}$

$\Delta S U V_{\text {mean }}$

$0,16^{\mathrm{c}}$

$\Delta$ SUV $_{\text {peak }}$

$0,87^{\mathrm{C}}$

$\triangle S U V_{\max } T B R$

$0,21^{\mathrm{c}}$

$\triangle \mathrm{SUV}_{\text {mean }} \mathrm{TBR}$

$0,26^{\mathrm{C}}$

$\triangle \mathrm{SUV}_{\text {peak }} \mathrm{TBR}^{\mathrm{R}}$

$0,59^{\mathrm{c}}$

Visual change

$0,69^{c}$

$\Delta \mathrm{SUV}_{\mathrm{max}}{ }^{\S}$ 


$$
\Delta \mathrm{SUV}_{\text {mean }} \S
$$

$\triangle$ SUV $_{\text {peak }} \S$

\section{$0,024^{\mathrm{C}}$}

aPearson Chi-Square, ${ }^{\text {b}}$ Fisher's Exact Test, ' ${ }^{\circ}$ Log Rank(Mantel-Cox), §if >30\%percent change accepted as significant

SUV max $_{\text {: }}$ maximum standard uptake value; SUV $_{\text {mean }}$ : mean standard uptake value; $S U V_{\text {peak: }}$ peak standard uptake value; TBR: tumor background rate.

\section{Figures}
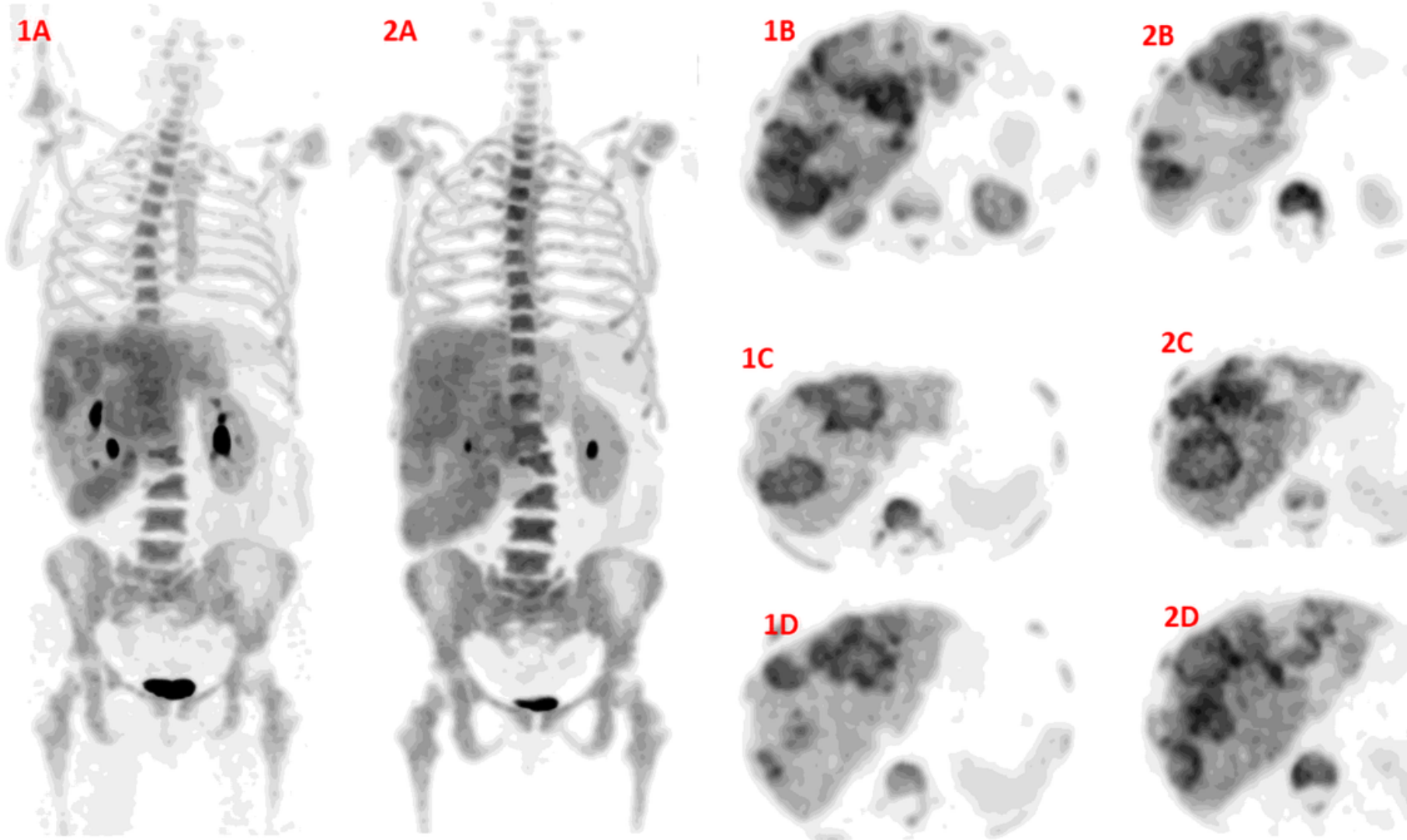

1C
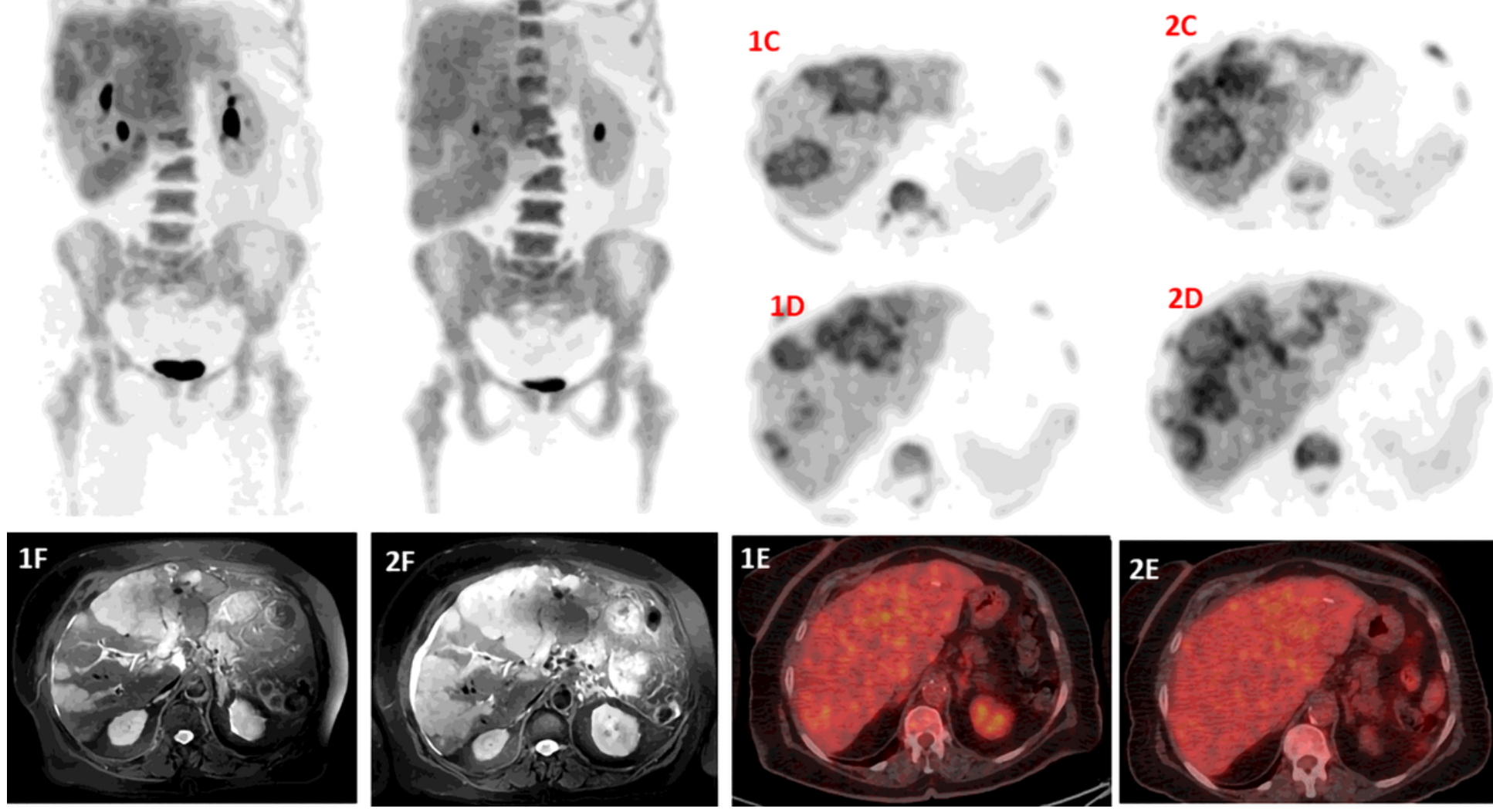

\section{Figure 1}

F18-FLT PET/CT MIP images before(1A) and after(2A) TARE therapy. Axial F18-FLT PET images revealing hypermetabolic lesions (SUVmax:16.1) before embolization (1B,1C,1D); post-therapy images revealed decreased activity on the left liver lobe, but most of the lesions were still metabolically active 
(SUVmax:16.4)(2B,2C,2D). Pre-treatment MR revealed multiple contrast-enhanced tumoral foci; after treatment, there were new lesions and progression on all lesions. T2-weighted MR shows multiple foci on both lobes before (1F) and after (2F) therapy. Tumoral foci were non-FDG-avid (1E) and did not change in avidity after TARE(2E).
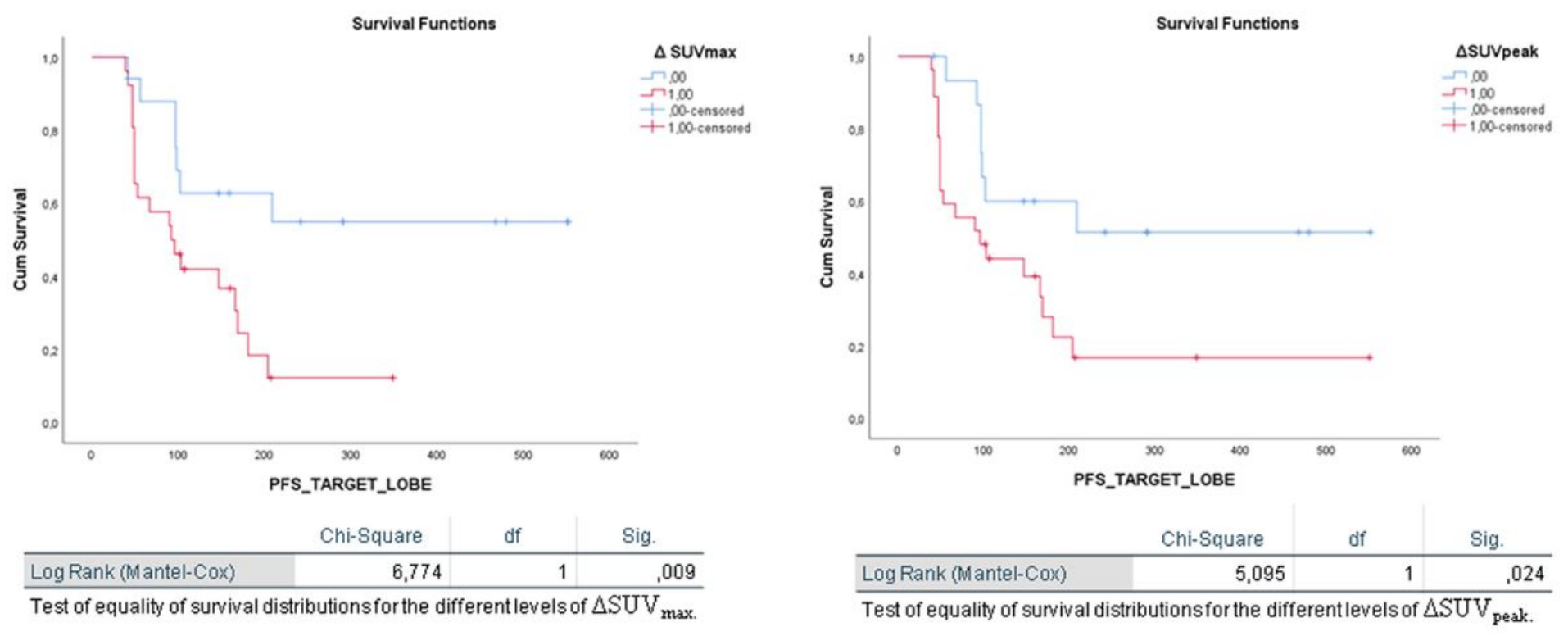

Figure 2

Kaplan-Meier method with log-rank test results revealing a significant difference in progression-free survival distribution for the $\Delta S U V \max (p<.009)$ and $\Delta \operatorname{SUVpeak}(p<.024)$ groups when cut-off $>30 \%$ change was applied.

\section{Supplementary Files}

This is a list of supplementary files associated with this preprint. Click to download.

- renamed0f856.pdf 